\title{
Spectroscopic modulation of multifunctionalized quantum dots for use as biological probes and effectors
}

\author{
Sandra Miskoski ${ }^{\mathrm{a}}$, Luciana Giordano ${ }^{\mathrm{a}}$, Maria H. Etchehon ${ }^{\mathrm{a}}$, Guillermo Menendez ${ }^{\mathrm{a}}$, \\ Keith A. Lidke ${ }^{\mathrm{b}, \mathrm{c}}$, Guy M. Hagen ${ }^{\mathrm{b}}$, Thomas M. Jovin ${ }^{\mathrm{b}}$ and Elizabeth A. Jares-Erijman ${ }^{1 \mathrm{a}}$ \\ ${ }^{a}$ Departamento de Química Orgánica, Facultad de Ciencias Exactas y Naturales, \\ Universidad de Buenos Aires, Ciudad Universitaria-Pabellón II, 1428 Buenos Aires, \\ Argentina; ${ }^{b}$ Department of Molecular Biology, Max Planck Institute for Biophysical \\ Chemistry, Am Fassberg 11, D-37077 Göttingen, Germany; ${ }^{\circ}$ Present address: Sandia \\ Laboratories, Albuquerque 87185, NM
}

\begin{abstract}
Multifunctional nanoparticles, quantum dots (QDs) are being developed as uniquely sensitive tools for elucidating the (bio)chemical and (bio)physical molecular mechanisms underlying functional states, i.e. the molecular physiology, of biological cells and organisms. Here we present a group of strategies and examples for (i) controlling the spectroscopic properties of QDs via Fluorescence Resonance Energy Transfer (FRET); (ii) determining the emission spectra of individual QDs in a population with an imaging spectrograph (ASI SpectraCube); and (iii) employing such liganded QDs as nano-probes in cellular studies of signal transduction.
\end{abstract}

Keywords: nanoparticle, quantum dot, photochromism, FRET, pcFRET, SpectraCube, EGF receptor

\section{INTRODUCTION}

Nanoparticles such as Quantum Dots (QDs) can be regarded as nanotools because they are scaled to molecular dimensions, offer the required spatial and temporal resolution, and incorporate spectroscopic tags, particularly fluorescent, providing the ultimate specificity and sensitivity. The nanoparticles are excellent probes for imaging in the context of the living cell, because only microscopy offers the multidimensional resolution (spatial, temporal, parametric) with which relevant correlations can be inferred. These considerations apply to normal cells as well as to those affected by stress or disease.

Quantum dots are generally capped by a polymeric layer required for solvent (aqueous) compatibility. This layer typically provides a high number of functional groups, which we have derivatized with molecules such as photochromic compounds or fluorophores providing control over the optical properties of the nanoparticle: emission intensity and spectrum and fluorescence lifetime. Specificity in targeting a specific protein or peptide is achieved by conjugation of ligands, generally in combination with expression tags.

\section{METHODOLOGY}

\subsection{FRET methods}

$R_{0}$, the critical Förster distance (for 50\% transfer efficiency), is defined by $R_{o}^{6}=8.785 \cdot 10^{-5} \kappa^{2} \Phi_{D} J n^{-4}$ (units, $\mathrm{nm}^{6}$ ), where $\Phi_{b}$ is the quantum yield of the donor in the absence of acceptor, $n$ is the refractive index of the medium, $\kappa^{2}$ is the orientation factor between donor and acceptor (here assigned the value of $2 / 3$ as generally, albeit often erroneously, assumed in the literature ${ }^{1}$ ), and $J$ is the spectral overlap integral between donor

1 eli@qo.fcen.uba.ar; phone/fax 0054-11-4576-3476

Colloidal Quantum Dots for Biomedical Applications, edited by Marek Osinski, Kenji Yamamoto, Thomas M. Jovin, Proc. of SPIE Vol. 6096, 60960X, (2006) · 0277-786X/06/\$15 - doi: 10.1117/12.649835 
emission and acceptor absorption, given by $J=\int F_{\lambda}^{D} \varepsilon_{\lambda}^{A} \lambda^{4} d \lambda$, where $F_{\lambda}$ is the normalized donor fluorescence spectrum and $\varepsilon_{\lambda}$ is the wavelength-dependent molar extinction coefficient $\left(\mathrm{M}^{-1} \mathrm{~cm}^{-1}\right)$ of the acceptor. For an isolated donor-acceptor pair, the FRET efficiency $E$ is given by $E=\left[1+\left(r / R_{o}\right)^{6}\right]^{-1}$ and for $n$ equivalent acceptors $E \approx\left[1+n\left(r / R_{o}\right)^{6}\right]^{-1}$.

\subsection{Steady-state and time-resolved spectroscopy}

Steady-state fluorescence measurements were performed with Perkin Elmer (Boston, MA) LS50B and SLM (Urbana, IL) $8000 \mathrm{~S}$ spectrofluorimeters at room temperature with $2 \mathrm{~nm}$ resolution. The light source for photochromic conversion was a Superlite SUV-DC-P system incorporating a $200 \mathrm{~W}$ DC Super-Pressure short arc lamp coupled to a light guide for high UV transmission (Lumatec GmbH, München, Germany). A UV bandpass filter (320-380 nm) was used for the photochromic forward conversion, and a green filter (510-580 $\mathrm{nm}$ ) for the backward conversion. The irradiance at the focused output of the light guide was $3.5 \mathrm{~mW} \mathrm{~cm}^{-2}$ at $365 \mathrm{~nm}$ and $20 \mathrm{~mW} \mathrm{~cm}$ at $546 \mathrm{~nm}$. Time resolved determinations were carried out in time correlated single photon counting mode using a Horiba Jobin Yvon (Edison NJ) TCSPC IBH spectrometer, with a nanoLED $373 \mathrm{~nm}$ source and a TBX-04 detector. Excitation was with a Omega (Bratteleboro, VT) BP370 DCF 10 and emission with a Schott (Iserlohn, Germany) OG515 longpass filter.

\subsection{Microscopy and hyperspectral imaging}

Imaging of QDs undergoing photochromic conversions was performed on an Olympus (Hamburg, Germany) IX71 inverted fluorescence microscope, equipped with a dual illumination system: (1) Hg arc lamp and filter selector $\left(365 \pm 10 \mathrm{~nm}\right.$, focal plane power and irradiance: $0.13 \mathrm{~mW}, 0.09 \mathrm{~W} \mathrm{~cm}^{-2} ; 546 \pm 10 \mathrm{~nm}$, focal plane power and irradiance: $11 \mathrm{~mW}, 8 \mathrm{~W} \mathrm{~cm}^{-2}$ ), and, alternatively, (2) a Cairn Research Ltd. (Faversham, U.K.) fiber optic coupled Optosource Xenon arc lamp source and P130 Optoscan monochromator $(450 \pm 15 \mathrm{~nm}$, focal plane power and irradiance: $0.4 \mathrm{~mW}, 0.03 \mathrm{~W} \mathrm{~cm}^{-2}$ ). Epi-illumination was with a 570 dichroic filter and the emission was collected with a Chroma Technology (Rockingham, VT) $565 \pm 10 \mathrm{~nm}$ filter optimized for the QD565-streptavidin conjugate quantum dots. The 60× 1.45 NA oil immersion objective had a field of view of $0.4 \mathrm{~mm}$. Images $(0.5 \mathrm{~s}$ exposure) were acquired with a Sensicam electron multiplying CCD camera (a kind loan of PCO, Kelheim, Germany) at an em gain of 50. Time series of 60 images ( $0.5 \mathrm{~s}$ exposures, $10 \mathrm{~s}$ intervals) were acquired during irradiation at 365 and $546 \mathrm{~nm}$. They were interleaved with single images excited at $450 \mathrm{~nm}$. Samples were prepared on poly-L-lysine pre-coated 18 well-flat $\mu$-slides (Integrated Biodiagnostics, München, Germany).

Hyperspectral imaging was performed with an Applied Spectral Imaging (Migdal Haemek, Israel) SpectraCube imaging spectrograph equipped with a VDS cool-1300 camera and mounted on the IX71 microscope. Quantum dot samples were imaged with a $150 \times 1.45 \mathrm{NA}$ objective. Excitation was with a Hg arc lamp and the epi-illumination filter Omega set consisted of $435 \mathrm{~nm}$ narrow bandpass excitation, $505 \mathrm{~nm}$ dichroic, and $510 \mathrm{~nm}$ longpass emission filters. Streptavidin-coated quantum dots (Quantum Dot Corp., Invitrogen, Carlsbad, CA) at 585, 605, or $655 \mathrm{~nm}$ were diluted from the original stock concentration to final concentrations of $1 \mathrm{pM}$ (QD655, PEG coated), $2 \mathrm{pM}$ (QD585), or $20 \mathrm{fM}$ (QD605) in PBS. Approximately $25 \mu 1$ of the diluted solution was pipetted onto an Ibidi (München, Germany) $18 \mu$-well, poly-L lysine coated slide and allowed to bind to the surface for $5 \mathrm{~min}$. The samples were washed once with water and maintained in water. A mixture of all 3 QD types was also prepared. Hyperspectral images in the range $500-800 \mathrm{~nm}$ were acquired in $185 \mathrm{~s}$, using 128 frames (1 s exposure) and 45 interferometer steps between each frame. After Hanning windowed-Fourier transformation, single QDs were marked and classified with the ASI SpectraView software. The edge of each full field image was selected as background, and the spectrum of this area was subtracted from the individual QD spectra.

\section{RESULTS AND DISCUSSION}

\subsection{Control of QD spectroscopic properties by FRET}




\subsubsection{Sensitized emission provides color changes}

Commercially available QDs bioconjugated to streptavidin and titrated with biotinylated fluorophores with suitable spectral properties can be quenched efficiently by FRET $^{2}$. Others have reported similar phenomena using QDs conjugated with maltose binding protein ${ }^{3-5}$.

In a recent study ${ }^{6}$, a color change of a biosensing QDs produced by binding of a fluorescent FRET acceptor served to determine the localization of the ectodomain of erbB1, the transmembrane receptor for epidermal growth factor (EGF), undergoing retrograde transport on cellular extensions known as filopodia after activation. Streptavidin conjugated QD525 nanoparticles carrying biotinylated EGF bound to and thereby activated erbB1. The cells were exposed to biotinylated Alexa546 (a potential FRET acceptor for the QD525) applied to the external medium and capable of binding to free streptavidin sites of accessible QDs. Two phenomena were observed, both indicating that the QD-receptor complexes remained externally located, i.e were not endocytosed (into the filopodia), during retrograde transport: (i) a quenching of the QD emission due to FRET; and (ii) sensitization via FRET, as well as direct excitation, of the Alexa546 emission, manifested as a change in apparent emission color of the QD-EGF-erbB1 complexes from green to yellowish-red (Fig. 1). Internalized QD-receptor complexes, not accessible to the Alexa546 ligand, retained their original spectral properties. Such a strategy not only serves to determine compartmentalization but also constitutes a means for selectively tagging a given QD subpopulation (see also Arndt-Jovin et al., this issue).
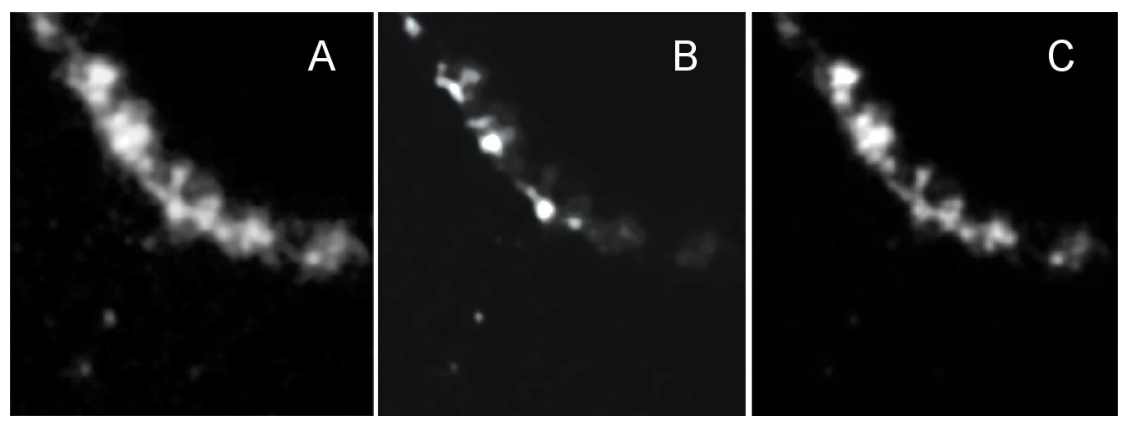

Figure 1. FRET-induced QD color change locates site of endocytosis of transporting EGF-QD525-EGFR complexes to the base of filopodia. A) b/w representation of all QDs on filopodia (dots along a diagonal) and on/in the cell (the arc delineates the cell surface with interior toward upper right) after addition of biocytin-Alexa546 as probe of external-internal compartmentalization (see text). B) yellowish-red emitting (due to FRET) QD525 subpopulation (filopodia and surface bound). C) green-emitting QD525 subpopulation, endocytosed prior to external addition of biocytin-Alexa546, and thus inaccessible. Adapted from ref. 6. Bar, $5 \mu \mathrm{m}$.

Two additional and potentially very useful manipulations of a QD:FRET-fluorophore system can be cited: (i) photobleaching the acceptor at a location and time of choice, thereby restoring the color of the targeted QDs and establishing a cohort that can be followed over time in the living cell; (ii) augmenting the luminosity of the nanoparticles by using photostable acceptors with lifetimes shorter than that of the QDs so as to increase the net radiative rate.

\subsubsection{Hyperpectral imaging of individual QDs with the SpectraCube imaging spectrograph}

The phenomena illustrated in the previous section imply the need for accurate spectral registration of individual QDs, or clusters thereof, particularly in cellular compartments. In order to take full advantage of the potential afforded by the unique characteristics of QDs (narrow emission spectra, broad excitation range, photostability), widefield imaging using systems that generate continuous emission spectra at every pixel position is highly desirable. Fourier encoding and CCD detection combines very efficient detection and spectral reconstruction of low-light level images. We demonstrate in this publication the first application, to our knowledge, of a commercial Fourier interferometric spectrograph, the SpectraCube manufactured by Applied Spectral Imaging, for spectral imaging of individual QDs. With the very high magnification afforded by a new unique $150 \times 1.45$ NA objective in the IX71 Olympus microscope, one obtains very distinct signals attributable to QDs either attached individually to a surface (Fig. 2) or on/in cells such as those in Fig. 1 (data not shown). In these preliminary measurements, the SpectraCube operational parameters were not yet 
optimized for QD detection in a systematic manner. Thus, we do not attribute the apparent dispersion in the spectra of each QD type in Fig. 2 solely to inherent properties of the QD preparations, which are presumed to be relatively monodisperse. Nonetheless, in the case of the mixed QD sample, the different types of QDs were easily distinguished by the SpectraCube, confirming that the resolved signals originated from individual nanoparticles and thus establishing that the spatial resolution and spectral sensitivity clearly suffice for single QD measurements. We are currently seeking to further expand the functionality of the SpectraCube by exploiting the single-photon sensitivity of electron multiplying CCD cameras, and by incorporating arbitrary spatial and spectral ROI selection, and a double-pass configuration enabling 2D (excitation, emission) spectra and optical sectioning?

\subsubsection{Modulation of QD emission intensity by pcFRET}

We have recently introduced the use of photochromic compounds as switchable acceptors for fluorescence resonance energy transfer (FRET) determinations according to a scheme we denote as photochromic FRET $(\text { pcFRET })^{8,9}$. This technique was developed to overcome limitations in the quantitative determination of FRET
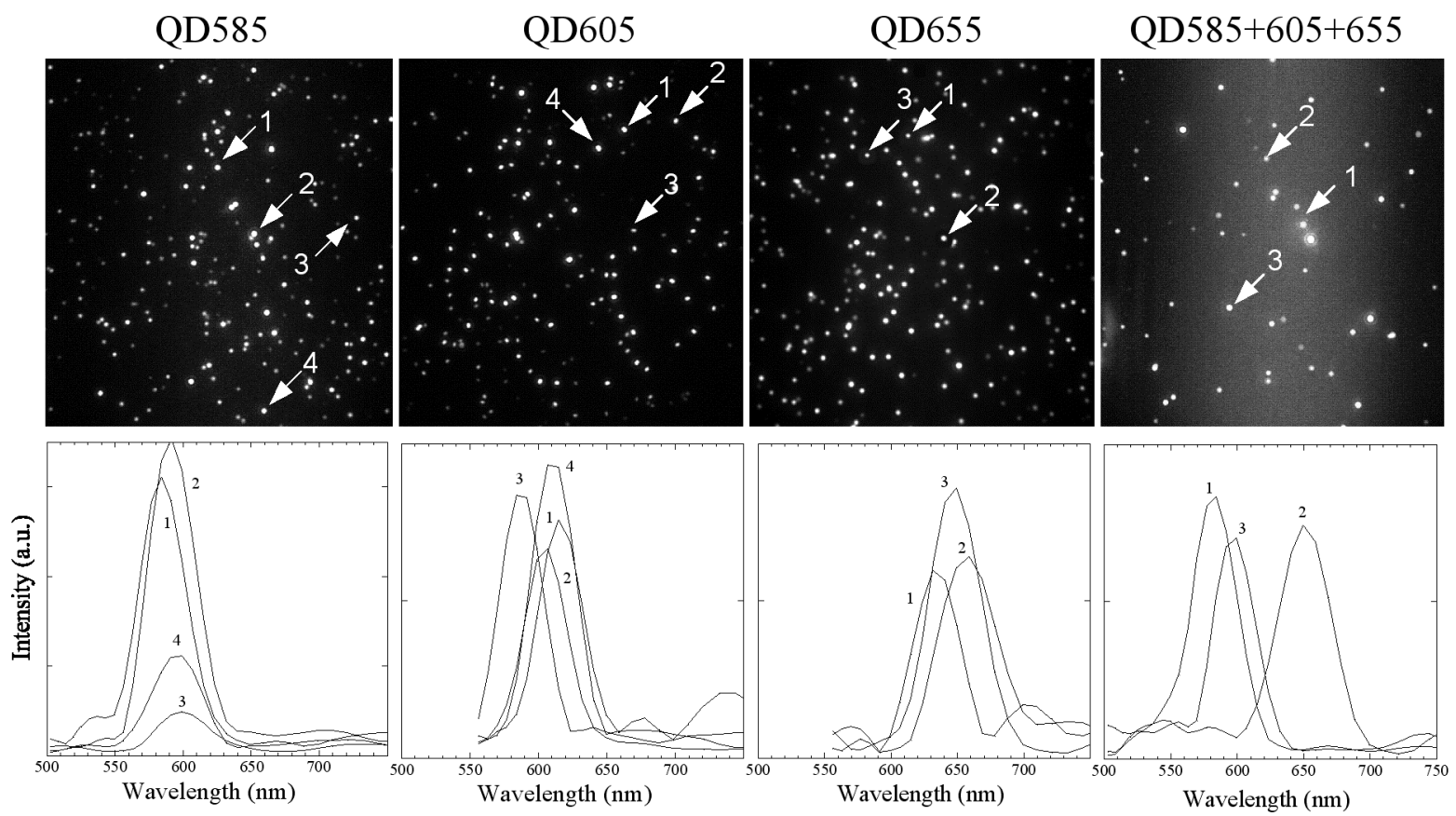

Figure 2. Hyperspectral imaging of QDs. Fields of individual QD preparations and their mixture (top row) and the associated spectra of selected QD positions (bottom row). Data acquired with the ASI SpectraCube. See text for details.

in vivo, particularly in imaging applications. In a recent review of this subject ${ }^{1}$ we classified pcFRET as a member of the family of so-called "acceptor depletion" methods by virtue of the induction of a reversible change in the structure and thus absorption properties of the photochromic compound (e.g. a diheteroarylethene) serving as the FRET acceptor. The key feature of pcFRET is the modulation of donor emission by cyclical transformations of the pc acceptor. The underlying phenomenon is the variation in the donor-acceptor overlap integral (and thus Förster transfer distance $R_{\mathrm{o}}$ ) between the two states; the FRET efficiency depends on the $6^{\text {th }}$ power of the relative separation $\left(r / R_{\mathrm{o}}\right)$ between the donor and acceptor. Nearultraviolet illumination leads to the conversion of the colorless open form to a closed form featuring an absorption band overlapping the emission band of the fluorescent donor, and thus to a quenching of the latter (FRET on state). Cycloreversion by exposure to visible light reverses the process, i.e. restores the FRET off state.

The reversible modulation of the emission of $\mathrm{CdSe} / \mathrm{ZnS}$ QDs was achieved by binding photochromic diheteroarylethenes and switchable acceptors for FRET (Fig. 3) ${ }^{10}$. A biotinylated diheteroarylethene derivative 
was bound to QDs bearing conjugated streptavidin, leading to an intensity decrease as a consequence of energy transfer to the closed form of the acceptor. Interconversion between the open and closed forms by irradiation with 365 and $546 \mathrm{~nm}$ light led to deactivation and activation, respectively, of the FRET process with a corresponding modulation of QD emission, observed both in solution and by sequential wide-field imaging (see Section 3.1.5).

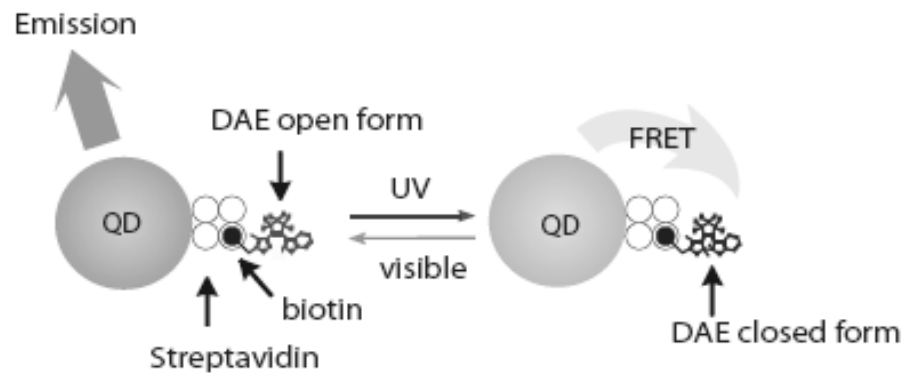

Figure 3. Schematic of pcFRET devices based on quantum dots QD-streptavidin with bound biotinylated diheteroarylethenes (DAE). Upon irradiation with UV light, the DAE, initially in the open colorless form, is converted to the closed colored form that functions as an FRET acceptor. Irradiation with visible light drives the DAE back to the open form, thereby deactivating the FRET process. In this manner, the emission properties of the QDs can be modulated by pcFRET.

\subsubsection{Selection of diheteroarylethene pcFRET acceptors}

We have recently synthetized ${ }^{11,12}$ several families of photochromic diheteroarylethenes bearing benzothiophene, thiophene and indole groups. The spectral characteristics of the closed forms were determined by the nature of the conjugation and substitution. The absorption spectra of compounds 1-5 (Fig. 4) are shown in Fig. 5. The selection of a photochromic acceptor is based on the overlap of its absorption bands with the emission of the QD donors. We have evaluated the characteristics of five comercial QDs (520, $565,585,605$ and $655 \mathrm{~nm}$ ) with a group of different photochromes (Table I).

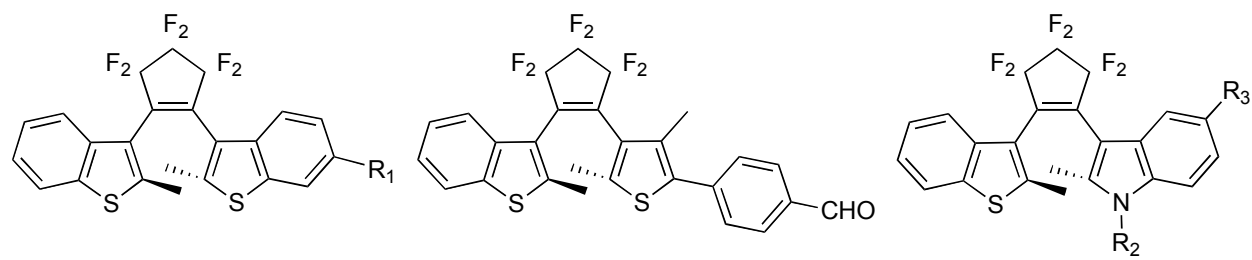

Figure 4. Photochromic compounds serve as pcFRET acceptors for QDs. Left structure: $1, \mathrm{R}_{1}: \mathrm{H} ; 2$, $\mathrm{R}_{1}=\mathrm{COOH}$; Middle structure: 3; Right structure: $4, \mathrm{R}_{2}=\mathrm{TBDMS}, \mathrm{R}_{3}=\mathrm{H} ; 5, \mathrm{R}_{2}=\mathrm{TBDMS}, \mathrm{R}_{3}=\mathrm{OMe}$.

The computed critical transfer distance $R_{\mathrm{o}}$ for the donor QDs 525, 565, 585, 605 and 655 and the acceptors open and closed forms of 5 different diheteroarylethene acceptors are given in Table I. The spectral absorption of the closed forms of the selected photochromic compounds span the emission region corresponding to the CdSe QDs. Spectral curves for the closed forms of compounds 1-5 are shown in Fig. 5B, together with the normalized emission spectra of the potential QD donors (Fig. 5A). Compound 1 and 2 have a maximum at 516 $\mathrm{nm}$, compounds 3 and 4 at 543 and $563 \mathrm{~nm}$, respectively, and compound 5, bearing a methoxy group in the indole ring, at $600 \mathrm{~nm}$. Compounds 1-4 possess appropriate properties for serving as switchable acceptors for QD520; i.e. $R_{\mathrm{o}}$ is $>3.5 \mathrm{~nm}$ in the closed forms and $<2.5 \mathrm{~nm}$ in the open forms. Similarly, compounds 1-5 can serve as acceptors for QD565. The spectral overlap is highest for compound 4, which has an $R_{\mathrm{o}}$ of $4.7 \mathrm{~nm}$. In the case of the QD655, the compound that provides the best overlap is number 5.

In the experiments featured below (Figs. 6 and 7), we selected as donor-acceptor pair QD565 and a derivative of compound 3 (Fig. 4) covalently bound to biotin. This acceptor quenched the QD emission by 70 $\%$, reflecting the large $R_{\mathrm{o}}\left(4.4 \mathrm{~nm}\right.$; overlap integral $\left.1.00 \cdot 10^{-13}\right)$ for the closed form of the diheteroarylethene. For the open form, $R_{\mathrm{o}}$ was $2.3 \mathrm{~nm}\left(J=2.11 \cdot 10^{-15}\right)$. The distance $r_{D A}$ between the center of the QD and the 
central atom of the acceptor was estimated (from data supplied by the manufacturers and the crystal structure of streptavidin) as $2.6-5 \mathrm{~nm}$; the range accounts for stereochemically feasible variations in the position of the four biotin binding sites of tetrameric streptavidin. Despite the uncertainty in $r_{D A}$, the computed FRET efficiency $E(0.71)$ clearly reflects primarily the process of FRET operating between the QD donors and the acceptors located on the surface. In fluorescence lifetime determinations (Fig. 6), QD565-ITK (QD preparations lacking surface PEG and thus better suited for FRET) displayed a decay that was fit to a biexponential function ( $\tau_{1}=20 \mathrm{~ns} ; 83 \%$ amplitude; $\tau_{2}=2.4 \mathrm{~ns}, 17 \%$ amplitude). After photoconversion pf the acceptor to the closed form, the steady-state intensity of the QD donor diminished by $68 \%$, paralleled by a corresponding reduction in the lifetimes $\left(\tau_{1}=9.5 \mathrm{~ns} ; 53 \%\right.$ amplitude; $\tau_{2}=1.75 \mathrm{~ns}, 47 \%$ amplitude). The efficiency $E$ corresponding to the open form was very low (0.05). The upper limit of $E$ (for the closed acceptor form) increases further in the case of multiple acceptors $(n>1)$ bound to the QD.

Table I. Overlap integrals and Förster distances for QD-pcFRET acceptors

\begin{tabular}{ccccccccccc}
\hline & \multicolumn{2}{c}{ QD525 } & \multicolumn{2}{c}{ QD565 } & \multicolumn{2}{c}{ QD585 } & \multicolumn{2}{c}{ QD605 } & \multicolumn{2}{c}{ QD655 } \\
\hline Cmpd & $J$ & $R_{\mathrm{o}}$ & $J$ & $R_{\mathrm{o}}$ & $J$ & $R_{\mathrm{o}}$ & $J$ & $R_{\mathrm{o}}$ & $J$ & $R_{\mathrm{o}}$ \\
$1 \mathrm{o}$ & $5.34 \cdot 10^{-16}$ & 1.8 & $3.66 \cdot 10^{-16}$ & 1.7 & $1.75 \cdot 10^{-16}$ & 1.5 & $2.4 \cdot 10^{-16}$ & 1.6 & $1.0 \cdot 10^{-15}$ & 2.1 \\
$1 \mathrm{c}$ & $5.36 \cdot 10^{-14}$ & 4.0 & $4.18 \cdot 10^{-14}$ & 3.8 & $1.64 \cdot 10^{-14}$ & 3.3 & $4.38 \cdot 10^{-15}$ & 2.6 & $5.9 \cdot 10^{-16}$ & 1.9 \\
$2 \mathrm{o}$ & $3.09 \cdot 10^{-16}$ & 1.7 & $3.48 \cdot 10^{-16}$ & 1.7 & $1.76 \cdot 10^{-16}$ & 1.5 & $3.34 \cdot 10^{-16}$ & 1.7 & $1.18 \cdot 10^{-15}$ & 2.2 \\
$2 \mathrm{c}$ & $5.55 \cdot 10^{-14}$ & 4.0 & $3.9 \mathrm{e} \cdot 10^{-14}$ & 3.7 & $1.06 \cdot 10^{-14}$ & 3.1 & $1.6 \cdot 10^{-15}$ & 2.2 & $4.06 \cdot 10^{-16}$ & 1.8 \\
$3 \mathrm{o}$ & $1.27 \cdot 10^{-15}$ & 2.1 & $2.11 \cdot 10^{-15}$ & 2.3 & $1.76 \cdot 10^{-15}$ & 2.3 & $1.56 \cdot 10^{-15}$ & 2.2 & $3.67 \cdot 10^{-15}$ & 2.6 \\
$3 \mathrm{c}$ & $6.40 \cdot 10^{-14}$ & 4.1 & $1.00 \cdot 10^{-13}$ & 4.4 & $8.81 \cdot 10^{-14}$ & 4.3 & $5.88 \cdot 10^{-14}$ & 4.1 & $1.73 \cdot 10^{-14}$ & 3.4 \\
$4 \mathrm{o}$ & $2.97 \cdot 10^{-16}$ & 1.7 & $6.60 \cdot 10^{-16}$ & 1.9 & $6.53 \cdot 10^{-16}$ & 1.9 & $5.31 \cdot 10^{-16}$ & 1.8 & $4.77 \cdot 10^{-16}$ & 1.9 \\
$4 \mathrm{c}$ & $6.71 \cdot 10^{-14}$ & 4.1 & $1.39 \cdot 10^{-13}$ & 4.7 & $1.44 \cdot 10^{-13}$ & 4.7 & $1.09 \cdot 10^{-13}$ & 4.5 & $2.92 \cdot 10^{-14}$ & 3.7 \\
$5 \mathrm{o}$ & $6.12 \cdot 10^{-17}$ & 1.3 & $1.81 \cdot 10^{-16}$ & 1.5 & $2.52 \cdot 10^{-16}$ & 1.6 & $3.3 \cdot 10^{-16}$ & 1.7 & $3.58 \cdot 10^{-16}$ & 1.8 \\
$5 \mathrm{c}$ & $2.52 \cdot 10^{-14}$ & 3.5 & $7.29 \cdot 10^{-14}$ & 4.2 & $1.01 \cdot 10^{-13}$ & 4.4 & $1.2 \cdot 10^{-13}$ & 4.6 & $8.96 \cdot 10^{-14}$ & 4.5 \\
\hline
\end{tabular}

o, c correspond to open and closed forms, respectively, of the photochromic acceptor. Quantum efficiencies were 0.4 for all QDs except for QD655 (0.5); values from Quantum Dot Corp. catalog. QD525 streptavidin conjugate, QD565-ITK streptavidin conjugate, QD585 streptavidin conjugate, QD605 streptavidin conjugate, QD655-biotin were used for spectral data collection (see also Fig. 5). The units of the overlap integral $J$ are $\mathrm{M}^{-1} \mathrm{~cm}^{-1} \mathrm{~nm}^{4}$ and of the Förster transfer distance $R_{\mathrm{o}}$, $\mathrm{nm}$.
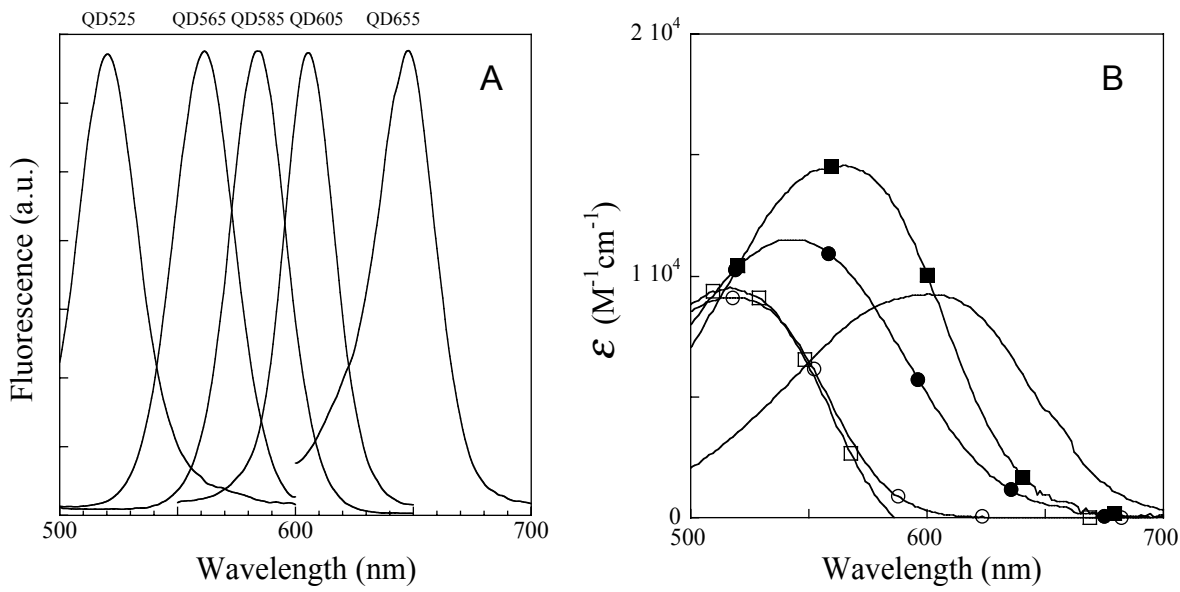

Figure 5. A) Normalized emission spectra of QD525, 565, 585, 605 and 655. B) Absorption spectra of compounds

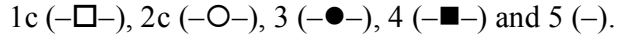



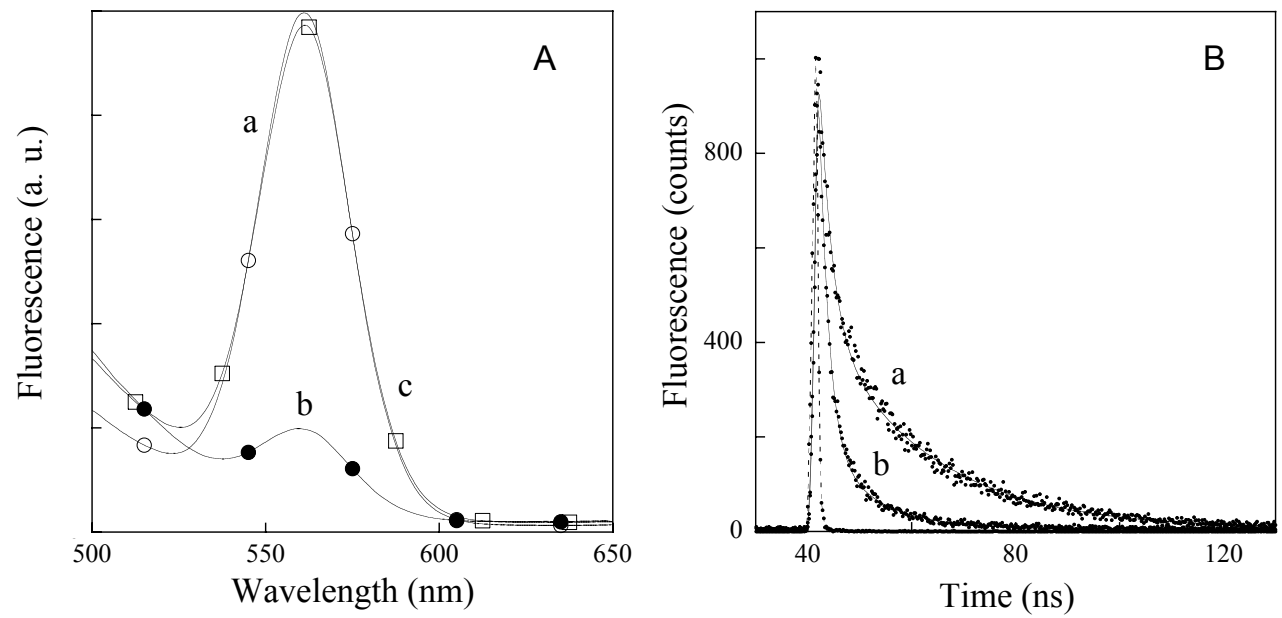

Figure 6. Changes in fluorescence intensity (A) and lifetime (B) accompanying photoconversion of biotinylated photochromic acceptor 3 complexed to QD565. Experimental conditions as in Section 2.2. a (-O-), open acceptor form; b(--), closed acceptor form; c (- - -), reconversion to the open form by irradiation with visible light.

\subsubsection{Cyclic modulation of QD donor fluorescence monitored by imaging}

Streptavidin-conjugated magnetic microspheres (Dynal, Invitrogen) were bound to polylysine-coated coverslips ( $\mu$-slides). The microspheres were then saturated with bis-biotin, washed, and exposed to a QD565streptavidin suspension. The $\mu$-slides were thereby covered with sparsely distributed $2.6 \mu \mathrm{m}$ magnetic beads, each of which was coated with QDs bridged to the carrier microspheres via bis-biotin, and by individual and clustered QDs spread randomly over the surface. After washing, the slides were exposed to saturating levels of photochromic compound 3 (Fig. 4).

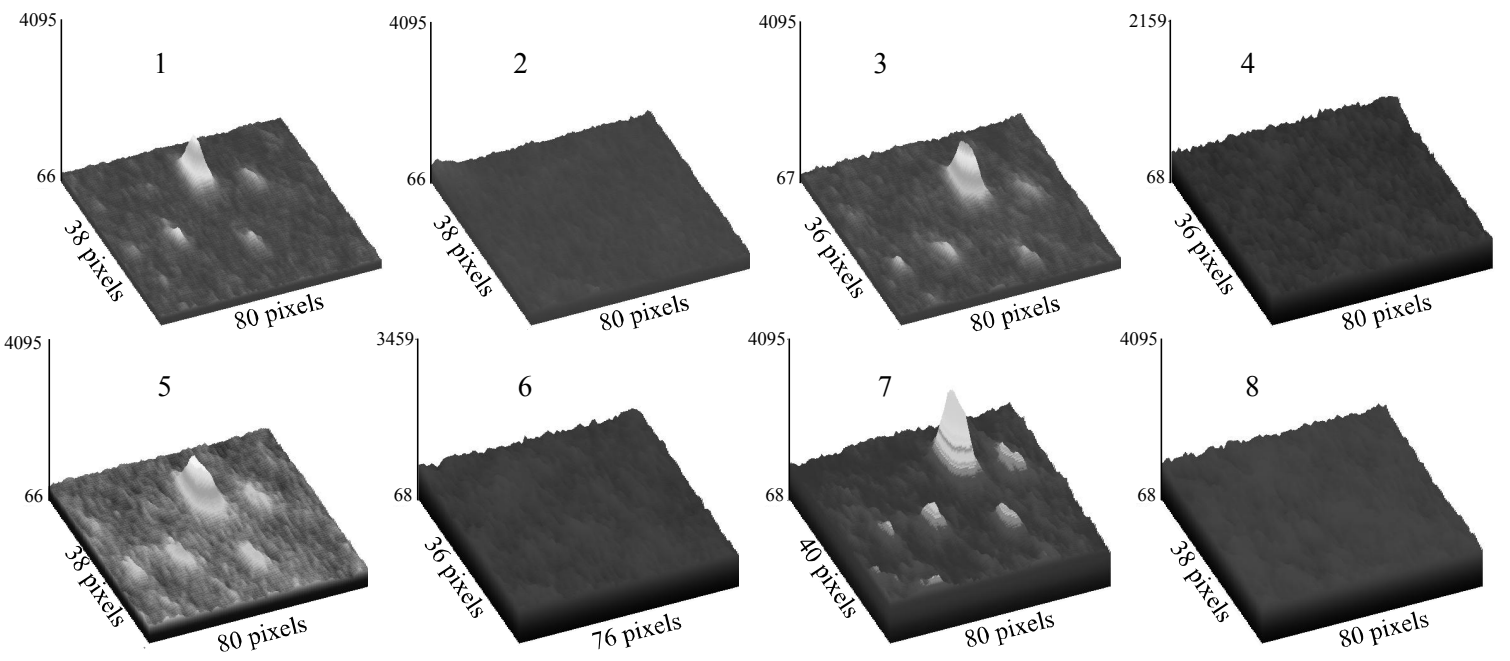

Figure 7. Imaging QD emission modulated by pcFRET. Streptavidin-conjugated magnetic microspheres were bound to polylysine-covered coverslips, washed with PBS $(20 \mathrm{mM} \mathrm{Na}$-phosphate, $150 \mathrm{mM} \mathrm{NaCl}, \mathrm{pH} 7.2)$ and incubated for $10 \mathrm{~min}$ with $2.6 \mu \mathrm{M}$ bis-biotin, $10 \mathrm{~min}$ with QD565-streptavidin in PBS, and 10 min with $2.5 \mu \mathrm{M}$ biotin-DAE in PBS; each incubation was followed by 3 washes with PBS. Surface plots of a selected region containing aggregates and single QDs. The surface exhibited fluorescence shells (QDs coated microspheres), and single and aggregated QDs. After a pre-irradiation period with UV and visible light the QDs displayed a reproducible decrease of emission upon irradiation with UV light (as a result of pcFRET) and an increase of emission when irradiated with visible light. 
The first exposure to UV light resulted in an increase of QD emission (representing a QD "curing" process), also observed in the spectroscopy measurements. After this equilibration phase, subsequent alternating exposures to UV and visible irradiation produced systematic decreases and increases, respectively, in QD emission. The latter achieved the same initial value after each cycle. We determined the kinetics of the forward conversion to the closed form and its cycloreversion under the given experimental conditions, from a series of images acquired during UV and visible irradiation. The kinetic curves were reproducible. Figure 7 features surface views corresponding to four cycles of photoconversion of a selected region. The initial and final states were monitored by imaging with low-level $450 \mathrm{~nm}$ excitation. The QD intensity exhibited a dramatic modulation, with an almost $100 \%$ decrease in emission intensity with the FRET-active state of the photochromic acceptor.

\section{AKNOWLEDGMENTS}

E.A. J.-E. and T.M. J. are recipients of grant I/77 897 from the Volkswagen Foundation. E.A. J.-E. is indebted to the Agencia Nacional de Promoción de la Ciencia y Tecnología (ANPCyT), Fundación Antorchas, Consejo Nacional de Investigaciones Científicas y Técnicas (CONICET), Secretaría de Ciencia, Tecnología e Innovación Productiva (SECyT), Germany-Argentine DLR-BMBF-SECyT, and the Universidad de Buenos Aires (UBA) for financial support. E.A. J.-E. is a Partner Group of the Max Planck Institute for Biophysical Chemistry, in Göttingen, Germany.

\section{REFERENCES}

1. E.A. Jares-Erijman and T.M. Jovin, "FRET imaging", Nat. Biotech., 21, 1387-1395, 2003.

2. H.E. Grecco et al., "Ensemble and single particle photophysical propeties (two-photon excitation, anisotropy, FRET, lifetime, spectral conversion) of commercial quantum dots in solution and in live cells", Microsc. Res. Tech., 65, 169-179, 2004.

3. I.L. Medintz et al., "Quantum dot bioconjugates for imaging, labelling and sensing", Nat. Mat., 4, 435446, 2005.

4. I.L. Medintz et al., "A fluorescence resonance energy transfer-derived structure of a quantum dot-protein bioconjugate nanoassembly", Proc. Nat. Acad. Sci. U.S.A., 101, 9612-9617, 2004.

5. I.L. Medintz et al., "A fluorescence resonance energy transfer sensor based on maltose binding protein", Bioconj. Chem., 14, 909-918, 2003.

6. D.S. Lidke et al., "Reaching out for signals: filopodia sense EGF and respond by directed retrograde transport of activated receptors", J. Cell Biol., 170, 619-626, 2005.

7. R. Heintzmann, K.A. Lidke, and T.M. Jovin, "Double-pass Fourier transform imaging spectroscopy". Optics Expr., 12: p. 753-763, 2004.

8. L. Song, E.A. Jares-Erijman, and T.M. Jovin, "A photochromic acceptor as a reversible light-driven switch in fluorescence resonance energy transfer (FRET)", J. Photochem. Photobiol. A-Chem., 150, 177$185,2002$.

9. L. Giordano, L., et al., "Diheteroarylethenes as thermally stable photoswitchable acceptors in photochromic fluorescence resonance energy transfer (pcFRET)", J. Am. Chem. Soc., 124, 7481-7489, 2002.

10. E.A. Jares-Erijman et al., "Imaging quantum dots switched on and off by photochromic fluorescence resonance energy transfer (pcFRET)", Molec. Cryst. Liq. Cryst., 430, 257-265, 2005.

11. L. Giordano, R.J. Vermeij, and E.A. Jares-Erijman, "Synthesis of indole-containing diheteroarylethenes. New probes for photochromic FRET (pcFRET)", Arkivoc Part xii, 268-281, 2005.

12. L. Giordano and E.A. Jares-Erijman: manuscript in preparation; L. Giordano, PhD Thesis, Univ. of Buenos Aires, 2006. 